\title{
Instrumentos, objetos e coleções como fontes para a história do ensino das ciências
}

\author{
Instruments, objects, and collections as sources \\ for the history of science education
}

\author{
Inês Gomes ${ }^{i}$ \\ ' Pesquisadora, Instituto de Ciências \\ Sociais/Universidade de Lisboa/ \\ Centro Interuniversitário de História \\ das Ciências e da Tecnologia. \\ Lisboa - Portugal \\ orcid.org/0000-0001-9210-9959 \\ gomes.ida@gmail.com
} Aprovado em 6 ago. 2018.
GOMES, Inês. Instrumentos, objetos e coleções como fontes para a história do ensino das ciências. História, Ciências, Saúde - Manguinhos, Rio de Janeiro, v.26, n.4, out.-dez. 2019, p.1211-1222.

\section{Resumo}

Um elevado número de escolas secundárias portuguesas possui coleções científicas, elementos centrais para a memória e identidade das instituições que as preservam, concorrendo para a compreensão das características que foram definindo a instrução pública de nível secundário em Portugal ao longo do tempo. Porém, esse património é ainda largamente desconhecido. $\mathrm{O}$ artigo chama a atenção para o debate historiográfico que envolve o uso de fontes materiais na história do ensino das ciências, dando a conhecer a relevância das coleções de história natural dos liceus de Portugal, bem como sua vulnerabilidade, dada a ausência de uma política geral ou diretrizes orientando sua conservação.

Palavras-chave: cultura material; história da ciência; história da educação; coleções de história natural; ensino secundário.

\section{Abstract}

A large number of Portuguese secondary schools have scientific collections, which are central elements for the memory and identity of the institutions that maintain them, and contribute to understanding the characteristics that over time have defined public secondary education in Portugal. However, this heritage is still largely unknown. This article draws attention to the historiographic debate surrounding the use of material sources in the history of science education, describing the relevance of natural history collections in Portuguese secondary schools as well as their vulnerability, considering the lack of a general policy or guidelines related to conservation.

Keywords: material culture; history of science; history of education; natural history collections; secondary education. 
Historiadores e sociólogos da educação enfatizam há muito que a pedagogia é tudo exceto uma atividade passiva ou neutra. O que conta como pedagogia 'apropriada' ou 'aceitável' num determinado contexto é sempre condicionado por decisões acerca das competências que os alunos devem adquirir (e porquê), e por preocupações relacionadas com o fornecimento de mão de obra e fluxo de capital humano para e além do contexto de ensino. As instituições educativas servem de cadinhos para a reprodução dos valores culturais, políticos e morais, bem como para a replicação de competências entre as novas gerações. As escolas orientam os alunos (com diferentes tons de subtileza e eficácia) para se tornarem bons cidadãos e forjarem identidades e papéis apropriados à sociedade. Claro, o que conta como 'boa cidadania', assim como o que conta como 'competências apropriadas', reflete sempre decisões ativas (e muitas vezes carregadas de controvérsias e amargas negociações) feitas em contextos específicos (Kaiser, 2005a, p.251; ênfases no original). ${ }^{1}$

Nos últimos anos, vários autores têm vindo a chamar a atenção para a necessidade de estudos que cruzem áreas de investigação como a história das ciências e a história da educação. No artigo "Training the generalist's vision in the history of science", acima citado, David Kaiser (2005a) trouxe, precisamente, à discussão o papel que o estudo da educação científica pode ter para a história, no seu sentido mais lato. Nos anos seguintes, outros historiadores reafirmaram a posição de Kaiser, chamando a atenção para a natureza fragmentada dos estudos históricos sobre educação das ciências (Rudolph, 2008) e defendendo que a "história da educação científica não é, nem pode mais ser, exterior à história das ciências" (Belhoste, 2011, p.51).

De fato, no campo da história das ciências, o estudo das práticas e contextos de produção de ciência tem sido privilegiado em face ao estudo das práticas e contextos da educação e disseminação. Ao mesmo tempo, na história da educação, o estudo das disciplinas da área das ciências tem sido tradicionalmente preterido ao estudo das disciplinas da área das humanidades. ${ }^{2}$ Todavia, nos últimos trinta anos, a investigação na área da história da educação das ciências tem vindo a intensificar-se e a diversificar-se, deixando de ser uma mera descrição cronológica centrada nos planos de estudo e nas instituições. ${ }^{3}$ Outros temas são, agora, valorizados: as relações entre os poderes político e económico e a educação; a formação das disciplinas; os manuais escolares; os exames; os professores; os alunos; a formação da persona científica e da comunidade científica; a "cultura escolar" (Molero Pintado, 2000); as aulas na sua prática quotidiana; entre outros. ${ }^{4}$ Em particular, a importância do estudo dos instrumentos e das coleções de ensino, ou por outras palavras, o papel das fontes materiais, como fonte para o conhecimento das práticas realizadas na sala de aula, bem como catalisador da aproximação entre história das ciências e história da educação, tem sido deveras enfatizado. ${ }^{5}$

Este artigo tem, precisamente, como objetivo chamar a atenção para a forma como, tanto a historiografia das ciências quanto a historiografia da educação se têm debruçado sobre a problemática das fontes materiais. Não se pretende ser exaustivo - nomeadamente por limitações de espaço -, mas antes dar conta do debate historiográfico que envolve o 
uso de instrumentos, objetos e coleções como fontes para a história do ensino das ciências. Por outro lado, pretende-se dar a conhecer, de uma forma geral, as coleções de ensino de história natural dos antigos liceus portugueses, enfatizando a sua relevância para a pesquisa sobre a história das ciências, assim como a sua vulnerabilidade, dada a ausência de uma política geral ou diretrizes orientando a sua conservação.

\section{Cultura material: a problemática das fontes materiais na história das ciências}

As fontes materiais são, na maioria das vezes, preteridas, privilegiando os historiadores o uso de fontes documentais textuais (Mosley, 2007). Tem-se vindo a reconhecer, no entanto, a importância das primeiras, a saber, a importância dos objetos, coleções, genericamente designados de "cultura material", para a história e, em particular, para a história das ciências:

O papel dos instrumentos mudou, é claro, à medida que a ciência mudou desde o século XVII, tanto em métodos quanto na sua organização social. Ao estudar instrumentos, podemos entender melhor como ocorreram as mudanças. ... Porque os instrumentos determinam o que pode ser feito, eles também determinam, até certo ponto, o que se pode pensar (Van Helden, Hankins, 1994, p.4).

O que nos dizem Albert van Helden e Thomas L. Hankins, por outras palavras, é que os objetos associados à produção e disseminação de conhecimento científico, no seu sentido mais lato (Warner, 1990), são evidências materiais de uma determinada comunidade ou sociedade. Eles corporizam importantes aspectos das práticas científicas, do conhecimento tácito, do desenvolvimento do inquiry experimental e da sua relação com a especulação teórica, da inovação e de múltiplas trading zones (cientistas, técnicos, preparadores, professores, estudantes, indústria, gestores, entre outros). ${ }^{6} \mathrm{Ou}$ seja, quando interpretados, os objetos permitem a compreensão do passado (Prown, 1996, p.25), isto é, promovem o enriquecimento da análise histórica, possibilitando olhares e perspectivas que sem a materialidade teriam sido ignorados (Gessner, 2012; Mosley, 2007).

É importante enfatizar que o significado de cultura material ultrapassa instrumentos e coleções, sendo um termo mais abrangente. Os próprios locais onde a ciência se desenvolve, produz e transmite são cruciais na determinação das práticas. ${ }^{7}$ Espaços e práticas estão indelevelmente ligados (Morus, 2010), influenciando o conhecimento que é produzido (Livingstone, 2003). É, por isso, de esperar, como nota Forgan (1986), que os edifícios onde se desenvolvem atividades científicas nos deem, também, informações valiosas sobre o desenvolvimento da ciência, bem como sobre a prática real da ciência. ${ }^{8}$

\section{Cultura material: a problemática das fontes materiais na história da educação}

No contexto da história da escola, ou seja, da história da educação, objetos, coleções e espaços são, também, considerados manifestações de práticas e saberes. Para Depaepe e Simon (1995, p.10), as fontes materiais constituem a "caixa negra" da história da educação, representando a "verdadeira atividade educativa" e permitindo, assim, aceder à "história da realidade educativa quotidiana". ${ }^{9}$ A história da escola já não se resume a estudos cronológicos 
da evolução do pensamento pedagógico, ou à análise das principais instituições dedicadas ao ensino. Uma nova história está hoje em marcha (Escolano Benito, 2000); uma história cultural e social da escola que tenta desvendar a "cultura escolar" (Molero Pintado, 2000). Por outras palavras, a história da escola procura descrever "um conjunto de "normas" que definem conhecimentos a ensinar e condutas a inculcar, e um conjunto de "práticas" que permitem a transmissão desses conhecimentos e a incorporação desses comportamentos" (Juliá, 2001, p.10), podendo, ainda, ser descrita como um conjunto de três culturas: a cultura empírico-prática, relacionada com a vida quotidiana das instituições; a cultura científica da educação, relacionada com as disciplinas académicas e a investigação educativa; e a cultura social e política, relacionada com os discursos e práticas de ordem político-institucional e que se expressa na linguagem normativa que serve de suporte à organização formal da educação (Escolano Benito, 2002, p.31-32). Nesse âmbito, um vasto conjunto de fontes deve ser levado em conta: textos pedagógicos, nomeadamente, em artigos de revistas especializadas, para aceder à cultura científica da educação; legislação, regulamentos e outros documentos administrativos, para aceder à cultura política; e cadernos, cartas, biografias, fotografias, exames, relatórios de professores, relatórios de inspetores, assim como mobília e materiais didáticos, para aceder à cultura empírico-prática. A história da educação passou, nesse contexto, a estudar as práticas, com enfoques etnográficos e micro-históricos (Escolano Benito, 2000, p.315, 317 e 319). Os objetos pedagógicos são sinais ou pegadas (Sacchetto, 1986, citado em Escolano Benito, 2007, p.19) que expressam características da escola (Escolano Benito, 2000, p.319); são marcas que podem informar sobre os "rituais" quotidianos (Hernández Díaz, 2002, p.225-226). ${ }^{10}$

Em particular, no âmbito do ensino das ciências, e apesar de a importância de se estudarem espaços e objetos ter sido assinalada há mais de trinta anos, ${ }^{11}$ só na última década o crescimento foi mais considerável. ${ }^{12}$ A materialidade e o património associados à escola foram objeto de vários números de revistas da especialidade; ${ }^{13}$ várias dissertações de mestrado e teses de doutoramento foram realizadas (Guerra, 2008; Lopes, 2004; Simón Castel, 2002; Gomes, 2014; Meloni, 2008); diversos livros foram publicados (Gaspar da Silva, Petry, 2012; López-Ocón, Aragón, Pedrazuela, 2012; Moreno Martínez, Sebastián Vicente, 2012) e vários congressos foram efetuados. ${ }^{14} \mathrm{O}$ livro Learning by doing: experiments and instruments in the history of science teaching é paradigmático dessa tendência, reunindo dois temas, nas palavras dos autores, nunca antes reunidos num único trabalho, (a) a prática experimental, instrumentos científicos e cultura material e (b) a história do ensino das ciências (Heering, Wittje, 2011, p.7). ${ }^{15}$ Começa, assim, a criar-se um corpo conceptual e metodológico considerável relativo à utilização das fontes materiais na história do ensino das ciências.

\section{As coleções de história natural dos antigos liceus portugueses}

O interesse em integrar a educação científica na narrativa da história das ciências, embora crescente, não tem incluído de igual forma os diferentes graus de ensino, centrando-se, a maioria dos estudos, no ensino superior. Se é verdade que é a esse nível que existe maior sobreposição entre os conteúdos e práticas de formação e de investigação (Kaiser, 2005b; 
Warwick, 2003; Kohler, 2008; Geison, 1981; Geison, Holmes, 1993), também é verdade que é o ensino das ciências no ensino secundário que permite que se desenvolvam vocações científicas. Nesse contexto, os séculos XIX e XX viram a promoção de um grande corpo de professores de ciências, sobre o qual repousa toda a estrutura institucional das comunidades científicas (Belhoste, jan. 1989, p.4). ${ }^{16}$ Por outro lado, o ensino das ciências ao nível do ensino médio reflete o papel social atribuído à ciência, uma vez que o seu objetivo transcende a preparação dos indivíduos para o ensino superior: ${ }^{17}$

se procurarmos considerar questões sobre a forma de como o conhecimento científico é apropriado e de como circula entre os cidadãos comuns, teremos dificuldade em encontrar um lugar mais adequado do que a sala de aula de ciências. A sala de aula é, afinal, um dos poucos lugares onde a ciência foi deliberadamente moldada para consumo público. Todo o cenário institucional da escolaridade formal - com suas leis de escolaridade obrigatória, instrução sistemática e expectativas de responsabilidade na aprendizagem dos alunos - dá à ciência escolar uma legitimidade que influencia poderosamente a forma como o público entende os conteúdos e os processos da ciência (Rudolph, 2005, p.357).

No que toca à cultura material do ensino secundário os trabalhos não têm sido de grande monta. No entanto, um olhar sobre, por exemplo, as coleções de história natural dos antigos liceus portugueses denota um património com um enorme potencial como fonte para o estudo da história do ensino das ciências.

Em Portugal, uma rede de escolas secundária surge a partir de 1836, num contexto em que a ideia de que eram necessárias instituições públicas e seculares para preparar os cidadãos para o ensino superior proliferava por toda a Europa, seguindo os exemplos franceses e alemães (Green, 2004; Anderson, 2004). No que diz respeito ao ensino das ciências, o primeiro decreto publicado reflete, claramente, o crescente interesse pela educação científica (Portugal, 17 nov. 1836). A ciência era fundamental para modernizar o país e desenvolver a nação. Consequentemente, o esforço para instituir disciplinas científicas no currículo da escola secundária foi simultâneo ao estabelecimento de uma rede escolar nacional de ensino médio. Ao mesmo tempo, as atividades práticas integraram, sempre, os currículos de ciências. Quando, em 1836, Passos Manuel determinou a criação da já mencionada rede de escolas secundárias, onde se lecionaria a cadeira de princípios de história natural dos três reinos da natureza aplicados às artes e ofícios, ordenou, também, a construção em cada um dos liceus de "um Jardim experimental destinado às aplicações de Botânica, e um gabinete que ter[ia] ... divisões correspondentes às aplicações ... da Zoologia e da Mineralogia" (Portugal, 17 nov. 1836, art. 68). Esses espaços criariam as necessárias condições para que o método indutivo pudesse ser devidamente aplicado. O ensino deveria ter por base a observação e partir do simples para o complexo e do concreto para o abstrato, sendo, dessa forma, prático e intuitivo. O gabinete ou museu constituía-se, então, como parte integrante e crucial para o ensino das ciências, e assim se manteve durante todo o século XIX e grande parte do século XX. O caráter descritivo dos programas e dos manuais fomentava, concomitantemente, a necessidade de se observarem os espécimes alvo de descrição. Aprender a classificar era um dos principais objetivos da aula. ${ }^{18} \mathrm{O}$ ensino dependia, assim, da realidade material do liceu. 
Gabinetes ou museus eram ferramentas indispensáveis e, nesse contexto, desde 1854 - quando as cadeiras de ciências foram efetivamente incluídas nos currículos dos liceus - chegaram às escolas de todo o país milhares de objetos. Ao longo dos anos, o governo assumiu protagonismo na estruturação da sala de aula, determinando os programas a lecionar e os métodos a utilizar. Todavia, a constituição de coleções de ensino envolveu um vasto conjunto de atores. O processo de construção da "sala de aula de ciências" requereu um esforço organizativo por parte do corpo docente. Os professores, nas relações que estabeleceram com outras instituições, com a sociedade civil e com o próprio Estado, tiveram um papel crucial como motor que desencadeou e permitiu a organização de coleções. Para aumentar as coleções das escolas de acordo com a política educativa, os professores encontraram alternativas ao Estado para o apetrechamento dos liceus. A Escola Politécnica, a Universidade de Coimbra, os Serviços Geológicos, alunos, ex-alunos, pais e "ilustres cidadãos" das comunidades onde os liceus se encontravam foram fontes fundamentais para a constituição, desenvolvimento e manutenção das coleções dos liceus. De um trabalho conjunto de deputados, ministros, governadores coloniais, professores universitários e professores de liceus, militares, pais de alunos e sociedade civil, organizaram-se os museus escolares, ordenados de acordo com os preceitos científicos que orientavam o ensino das ciências (Gomes, 2014, p.121-186).

Não se conhece, ao certo, o número de coleções de ensino de história natural - associadas aos antigos liceus - que existem, atualmente, em Portugal. Em 1978, quando os liceus foram extintos, passando a designar-se escolas secundárias, a sua maioria possuía coleções para o ensino prático das ciências naturais. Tendo em conta que a passagem de liceu para escola secundária foi apenas nominal, não tendo acarretado mudanças de instalações, as escolas secundárias do pós-25 de abril receberam das suas predecessoras um conjunto de materiais, nomeadamente as coleções de história natural, sendo de esperar que muitas dessas coleções ainda existam, podendo constituir-se como elementos centrais para a memória e identidade das instituições que as possuem. No entanto, ninguém sabe com rigor o que existe, dado que este património tem permanecido desconhecido e inacessível.

Apesar do vasto património das escolas secundárias portuguesas no que concerne ao ensino das ciências naturais - animais naturalizados e conservados em líquido; coleções osteológicas, herbários; rochas, minerais e fósseis; modelos anatómicos de animais e plantas; modelos cristalográficos; microscópios e lupas e quadros parietais -, parte do qual fabricado no século XIX e início do século XX, podendo ser, por vezes, de épocas anteriores, e sendo alguns provenientes de importantes fabricantes europeus, como as casas Émile Deyrolle, Robert Brendel ou Louis Auzoux, o trabalho de base - a sua inventariação, preservação e acessibilidade, bem como da documentação e arquivos a ele associados - encontra-se, ainda, por fazer.

$\mathrm{Na}$ verdade, as coleções de história natural das escolas secundárias portuguesas encontram-se numa situação de semiabandono, como consequência do declínio do seu uso nas aulas, da falta de pessoal, da falta de tempo e da falta de conhecimento dos responsáveis. As condições de acondicionamento dessas coleções não são as melhores: os exemplares são, muitas vezes, guardados em caixotes de cartão em sótãos e armazéns, revelando sinais de deterioração e contaminação por micro-organismos. Ao mesmo tempo, na maioria das 
escolas não existem inventários completos ou catálogos do material, bem como estudos sobre as suas coleções. Por um lado, os exemplares não se encontram identificados quanto à taxonomia, origem geográfica ou data de colheita, o que obriga ao cruzamento com outros documentos históricos para a recuperação da história da coleção e dos dados associados. Por outro, o seu estudo envolve ainda um vasto leque de informação reunida em livros de texto, inventários, legislação escolar, apontamentos de professores, cadernos de alunos, catálogos de fabricantes, registo de compras, livros de contabilidade, correspondência, anuários, exames etc. (Simón Castel, 2008; Simon, 2006; Mogarro, 2006; Simon, Bertomeu Sánchez, García Belmar, 2009). Porém, na maioria das escolas visitadas, as coleções de história natural não têm arquivos ou documentação associada. Em suma, há nas escolas secundárias portuguesas, de uma forma geral, um desconhecimento profundo da importância desse património e, sobretudo, das práticas adequadas à preservação desses materiais.

Todavia, e apesar desse cenário, alguns estudos recentes apontam para o fato de essas coleções concorrerem para a compreensão das características que definem a instrução pública de nível secundário em Portugal, durante o século XIX e grande parte do século XX (Lopes, 2004; Guerra, 2008; Leal, 2007; Gomes, 2014, 2017, 2018). Essas coleções são evidência da forma como a ciência foi ministrada, assim como do seu estatuto no seio do ensino, materializando de forma sistemática e coerente a evolução do ensino científico em Portugal. As coleções dos antigos liceus não são, apenas, "objetos empoeirados", constituindo, antes, um património científico rico, nacionalmente distribuído e com significado contemporâneo (Gomes, 2014, p.67). Mesmo reconhecendo a relevância desse património, colocam-se ainda múltiplos desafios no que diz respeito à sua preservação e acessibilidade. Desde logo desafios institucionais, nomeadamente problemas financeiros, de pessoal qualificado e de espaço. É preciso, urgentemente, conhecer e estudar o que existe (Gomes, 2014, p.68).

\section{Considerações finais}

No âmbito da renovação dos estudos sobre história da educação das ciências a que atualmente se assiste, a história das ciências e a história da educação cruzam-se inevitavelmente. Segundo as novas perspectivas, a diferença entre o discurso normativo e a realidade é grande, sendo necessário olhar para novos elementos que reflitam o quotidiano, em que os objetos, as coleções e os espaços assumem um papel de grande relevância.

Estudos que considerem as coleções de ensino de ciências - na sua constituição, desenvolvimento, uso e até desmantelamento e dispersão - como elementos de análise centrais para uma compreensão mais rica das práticas e contextos locais e globais de produção e disseminação da ciência são urgentes. Essas abordagens, ainda pouco frequentes nos estudos históricos, quer em Portugal quer no nível internacional, tornam-se ainda mais pertinentes quando investigações recentes apontam para o fato de, ao conhecer a história desse património, serem revelados novos aspectos relativos ao ensino das ciências e ao uso de instrumentos e coleções nas salas de aula (Gomes, 2018).

Finalmente, é importante notar a relevância capital da catalogação de coleções. É urgente ter uma ideia mais clara de como é constituído, exatamente, o património científico 
português das escolas de ensino médio. Para além do mais, é imperativo que se definam políticas e diretrizes para a catalogação, conservação, restauração, armazenamento, promoção, estudo e reconhecimento desse tão rico património científico.

\section{AGRADECIMENTOS}

Este trabalho foi desenvolvido no Centro Interuniversitário de História das Ciências e da Tecnologia e financiado por Fundos Nacionais por meio da Fundação para a Ciência e a Tecnologia (FCT) no âmbito dos projectos SFRH/BD/47653/2008 e UID/HIS/00286/2013. Gostaria, também, de agradecer ao Luís Vicente, que me "apresentou" os museus escolares de história natural, e à Marta Lourenço, que acolheu desde o primeiro momento o meu projeto de investigação sobre eles, e cujas contribuições para a sua génese e desenvolvimento foram essenciais. Gostaria, ainda, de agradecer à Ana Nunes pelo apoio na elaboração deste artigo.

\section{NOTAS}

${ }^{1}$ Nesta e nas demais citações de textos publicados em outros idiomas, a tradução é livre.

${ }^{2}$ Algumas exceções são, por exemplo, Rudolph $(2002,2005)$ e Belhoste (jan. 1989).

${ }^{3}$ Brock (1975) e Rudolph (2008) fizeram uma revisão bibliográfica da investigação desenvolvida na área da história da educação das ciências.

${ }^{4}$ Entre outros, ver: Rudolph (2002, 2005); Kohlstedt (1988, 1990, 2005); Kaiser (2005b); Simon (2008); Warwick (2003); Kohler (2011); Simon, Herran (2008); Simon, Bertomeu Sánchez, García Belmar (2009).

${ }^{5}$ Recentemente, Bertomeu Sánchez e Simón Castel (2012) vieram sistematizar, de forma clara, a importância da gradual aproximação entre história das ciências e história da educação, valorizando, de resto, o papel das fontes materiais como catalisador dessa démarche.

${ }^{6}$ Mosley (2007) vai mesmo mais longe, afirmando que o próprio desenho, construção e produção de instrumentos científicos faz, também, parte do "processo de conhecimento científico".

${ }^{7}$ Os estudos de Hannaway (1986) ou Shapin (1988) são alguns exemplos que deixam claro o poder identitário dos espaços científicos. O número da revista Isis onde foi publicado o artigo citado de Shapin foi inteiramente dedicado à "prática científica in situ". Ver também, por exemplo, García Belmar e Bertomeu Sánchez (2009).

${ }^{8}$ Ver também Kohler (2008).

9 Bertomeu Sánchez e García Belmar (2002, p.11) designam precisamente de "caixas negras" a coleção de instrumentos científicos da Universidade de Valência, explicando: "Abrir las cajas negras implica una reflexión sobre los usos de los instrumentos científicos antiguos como fuentes para la historia de la ciencia como instrumentos didácticos al servicio de la enseñanza de las ciencias y como objetos museográficos con una enorme potencialidad en la divulgación científica".

${ }^{10}$ Ver também Grosvenor, Lawn, Rousmaniere (1999).

${ }^{11}$ Brock (1975, p.73-74) chamava a atenção de que o ensino prático das ciências implicava o uso de aparelhos e instrumentos e que a sua utilização envolvia a adaptação de salas e a construção de laboratórios. Este era, para Brock, um assunto que "merecia investigação" e que, até a data, tinha sido um "tópico negligenciado". O autor referiu-se, mesmo, aos "aparelhos 'históricos' sobreviventes" das escolas de Yorkshire, considerando imprescindível que se visitassem as escolas antigas e se catalogassem os seus instrumentos.

${ }^{12} \mathrm{Em}$ 2008, Rudolph notava que estudos recentes com recurso a fontes materiais começavam, então, a ajudar a perceber o papel dos objetos, instrumentos e coleções no ensino das ciências, citando apenas investigações realizadas a partir dos finais da década de 1990: Bucchi (1998), Morus (2006).

${ }^{13}$ Pro-Posições (Cultura escolar..., 2005); Revista Brasileira de História da Educação (Arquivos, 2011; A cultura..., 2007); Participación Educativa (História..., mar. 2008).

${ }^{14} \mathrm{O}$ nono Congresso Luso-brasileiro de História da Educação foi subordinado ao tema "Rituais, espaços e patrimónios escolares", e o terceiro Foro Ibérico de Museísmo Pedagógico - quintas Jornadas Científicas de la Sociedad Española para el Estudio del Patrimonio Histórico Educativo -, dedicado ao "Patrimonio y etnografía de la escuela en España y Portugal durante el siglo XX".

${ }^{15}$ Sobre a mesma temática, ver o número especial da revista Science and Education (The history..., 2012), cujo tema é "The history of experimental science teaching". 
${ }^{16}$ Ver também Kohlstedt (2005, p.325).

${ }^{17}$ A relevância do estudo do ensino secundário - não exclusivamente do ensino das ciências - foi, também, realçada pela revista Paedagogica Historica (2004), que dedicou um número, precisamente, à história institucional, cultural e social da educação secundária.

${ }^{18}$ Gomes (2014, p.71-120) e Leal (2007, p.149-168) sumarizam os planos de estudo dos liceus entre 1836 e 1975 e 1895 e 1954 respetivamente.

\section{REFERÊNCIAS}

A CULTURA...

A cultura material na história da educação: possibilidades de pesquisa. Revista Brasileira de História da Educação, v.7, n.2 [14]. [Dossiê]. Disponível em: <http://periodicos.uem.br/ojs/ index.php/rbhe/issue/view/1378/showToc>. Acesso em: 19 jul. 2019. 2007.

ANDERSON, Robert.

The idea of the secondary school in nineteenthcentury Europe. Paedagogica Historica, v.40, n.12, p.93-106. 2004.

\section{ARQUIVOS...}

Arquivos, objetos e memórias educativas: práticas de inventário. Revista Brasileira de História da Educação, v.11, n.1 [25]. [Dossiê]. Disponível em: <http://periodicos.uem.br/ojs/ index.php/rbhe/issue/view/1369/showToc>. Acesso em: 19 jul. 2019. 2011.

BELHOSTE, Bruno.

Das ciências instituídas às ciências ensinadas, ou como levar em conta a atividade didática na história das ciências. Revista Brasileira de História da Educação, v.11, n.3, p.47-61. 2011.

BELHOSTE, Bruno.

Les caractères généraux de l'enseignement secondaire scientifique: de la fin de l'Ancien Régime à la Première Guerre mondiale. Histoire de l'Education, n.41, p.3-45. jan. 1989.

BERTOMEU SÁNCHEZ, José Ramón; GARCÍA BELMAR, Antonio (Ed.).

Abriendo las cajas negras: los instrumentos científicos de la Universidad de Valencia. Valencia: PUV. 2002.

\section{BERTOMEU SÁNCHEZ, José Ramón; SIMÓN} CASTEL, Josep.

Viejos objetos y nuevas perspectivas historiográficas: la cultura material de la ciencia en las aulas del siglo XIX. In: López-Ocón, Leoncio; Aragón, Santiago; Pedrazuela, Mario (Ed.). Aulas con memoria: ciencia, educación y patrimonio en los institutos históricos de Madrid (1837-1936). Madrid: Ciencia y Educación en los Institutos Madrileños de Enseñanza Secundaria (Ceimes); Doce Calles; Comunidad de Madrid. p.49-72. 2012.
BROCK, William H.

From Liebig to Nuffield: a bibliography of the history of science education, 1839-1974. Studies in Science Education, v.2, n.1, p.67-99. 1975.

BUCCHI, Massimiano.

Images of science in the classroom: wallcharts and science education 1850-1920. The British Journal for the History of Science, v.31, n.2, p.161-184. 1998.

CULTURA ESCOLAR...

Cultura escolar e cultura material escolar: entre arquivos e museu. Pro-Posições, v.16, n.1 [Dossiê]. Disponível em: <https://www.fe.unicamp.br/ publicacoes/lancamentos/2304>. Acesso em: 19 jul. 2019. 2005.

DEPAEPE, Marc; SIMON, Frank.

Is there any place for the history of "education" in the "history of education"? A plea for the history of everyday educational reality in and outside schools. Paedagogica Historica, v.31, n.2, p.9-16. 1995.

ESCOLANO BENITO, Agustín.

La cultura material de la escuela. In: Escolano Benito, Agustín (Ed.). La cultura material de la escuela: en el centenario de la Junta para la Ampliación de Estudios, 1907-2007. Berlanga de Duero: Centro Internacional de Cultura Escolar. p.15-24. 2007.

ESCOLANO BENITO, Agustín.

Memoria de la educación y cultura de la escuela. In: Escolano Benito, Agustín; Hernández Díaz, José María (Ed.). La memoria y el deseo: cultura de la escuela y educación deseada. Valencia: Tirant lo Blanch. p.19-42. 2002.

ESCOLANO BENITO, Agustín.

La historia de la educación después de la posmodernidad. In: Ruiz Berrio, Julio (Ed.). $\mathrm{La}$ cultura escolar de Europa: tendencias históricas emergentes. Madrid: Biblioteca Nueva. p.297323. 2000.

FORGAN, Sophie.

Context, image and function: a preliminary enquiry into the architecture of scientific societies. The British Journal for the History of Science, v.19, n.1, p.89-113. 1986. 
GARCÍA BELMAR, Antonio; BERTOMEU SÁNCHEZ, José Ramón.

Teaching and research spaces: the chemistry chair of the Collège de France, 1770-1840. In: Lourenço, Marta C.; Carneiro, Ana (Ed.). The Laboratorio Chimico Overture: spaces and collections in the history of science, Lisbon. Lisboa: Museu de Ciência da Universidade de Lisboa (MCUL). p.33-54. 2009.

GASPAR DA SILVA, Vera Lucia; PETRY, Marilia Gabriela (Ed.).

Objetos da escola: espaços e lugares de constituição de uma cultura material escolar (Santa Catarina, séculos XIX e XX). Florianópolis: Insular. 2012.

GEISON, Gerald L.

Scientific change, emerging specialties, and research schools. History of Science, v.19, n.1, p.20-40. 1981.

GEISON, Gerald L.; HOLMES, Frederic L. (Ed.). Research schools: historical reappraisals. Osiris, v.8 (Thematic issue), p.I-VIII; 1-248. 1993.

GESSNER, Samuel.

Through the filter of history: how early instruments in Portugal bear witness to mathematical culture around 1600. Trabalho apresentado no eighth Science and Technology in the European Periphery (Step) Meeting, 2012. Corfu. 2012

GOMES, Inês.

The scientific heritage of Portuguese secondary schools: a historical approach. Paedagogica Historica, v.54, n.4, p.468-484. 2018.

GOMES, Inês.

The natural history collection at the Lisbon Military College: tracing the history of a teaching collection. Journal of the History of Collections, v.29, n.3, p.409-422. 2017.

GOMES, Inês.

Os museus escolares de história natural: análise histórica e perspectivas de futuro (1836-1975). Dissertação (Doutorado em História e Filosofia das Ciências) - Universidade de Lisboa, Lisboa. 2014.

GREEN, Andy.

Education and state formation: the rise of education systems in England, France and the USA. London: Macmillan. 2004.

GROSVENOR, Ian; LAWN, Martin; ROUSMANIERE, Kate (Ed.).

Silence and images: the social history of the classroom. New York: Peter Lang. 1999.

GUERRA, Marcolina.

Memória e materialidade no ensino liceal: um percurso pelo património e materiais didácticos do Liceu de Portalegre. Dissertação (Mestrado em Ciências da Educação) - Universidade de Lisboa, Lisboa. 2008.

HANNAWAY, Owen.

Laboratory design and the aim of science: Andreas Libavius versus Tycho Brahe. Isis, v.77, n.4, p.585-610. 1986.

HEERING, Peter; WITTJE, Roland.

Introduction: neglected uses of instruments and experiments. In: Heering, Peter; Wittje, Roland (Ed.). Learning by doing: experiments and instruments in the history of science teaching. Stuttgart: Franz Steiner. p.7-13. 2011.

HERNÁNDEZ DÍAZ, José María.

Etnografía e historia natural de la escuela. In: Escolano Benito, Agustín; Hernández Díaz, José María (Ed.). La memoria y el deseo: cultura de la escuela y educación deseada. Valencia: Tirant lo Blanch. p.225-246. 2002.

HISTORIA...

Historia de un olvido: patrimonio en los centros escolares. Participación Educativa, v.7 [Número temático]. Disponível em: <https://www. educacionyfp.gob.es/dam/jcr:91164978-14a740c7-8c44-0467ae763e4c/07_marzo_2008.pdf >. Acesso em: 19 jul. 2019. mar. 2008.

JULIÁ, Dominique.

A cultura escolar como objeto histórico. Revista Brasileira de História da Educação, v.1, n.1, p.9-43. 2001.

KAISER, David.

Training the generalist's vision in the history of science. Isis, v.96, n.2, p.244-251. 2005a.

KAISER, David (Ed.).

Pedagogy and the practice of science: historical and contemporary perspectives. Cambridge: The MIT Press. 2005b.

KOHLER, Robert E.

Os senhores da mosca: a genética drosophila e a vida experimental. Porto: Porto Editora. 2011.

KOHLER, Robert E.

Lab history: reflections. Isis, v.99, n.4, p.761-768. 2008.

KOHLSTEDT, Sally Gregory.

Nature, not books: scientists and the origins of the nature study movement in the 1890s. Isis, v.96, n.3, p.324-352. 2005.

KOHLSTEDT, Sally Gregory.

Parlors, primers, and public schooling: education for science in nineteenth-century America. Isis, v.81, n.3, p.424-445. 1990.

KOHLSTEDT, Sally Gregory. Curiosities and cabinets: natural history museums and education on the antebellum campus. Isis, v.79, n.3, p.405-426. 1988. 
LEAL, Catarina.

Na sombra da história natural: o ensino liceal das ciências biológicas e geológicas (1895-1954). Dissertação (Mestrado em Ciências da Educação) - Universidade de Lisboa, Lisboa. 2007.

LIVINGSTONE, David N.

Putting science in its place: geographies of scientific knowledge. Chicago: Chicago University Press. 2003.

LOPES, Rui.

Captar a atenção, ilustrar a memória! Viagem ao universo de mapas e outras imagens parietais do Liceu de Passos Manuel. Dissertação (Mestrado em Ciências da Educação) - Universidade de Lisboa, Lisboa. 2004.

LÓPEZ-OCÓN, Leoncio; ARAGÓN, Santiago; PEDRAZUELA, Mario (Ed.).

Aulas con memoria: ciencia, educación y patrimonio en los institutos históricos de Madrid (1837-1936). Madrid: Ciencia y Educación en los Institutos Madrileños de Enseñanza Secundaria (Ceimes); Doce Calles; Comunidad de Madrid. 2012.

MELONI, Reginaldo.

Saberes em ciências naturais: o ensino da física e química no Colégio Culto à Ciência, 1873-1910. Tese (Doutorado em História da Educação) Universidade de Campinas, Campinas. 2008.

MOGARRO, Maria João.

Arquivo e educação: a construção da memória educativa. Sísifo: Revista de Ciências da Educação, n.1, p.71-84. set.-dez. 2006.

MOLERO PINTADO, Antonio.

En torno a la cultura escolar como objeto histórico. In: Ruiz Berrio, Julio (Ed.). La cultura escolar de Europa: tendencias históricas emergentes. Madrid: Biblioteca Nueva. p.223228. 2000.

MORENO MARTÍNEZ, Pedro L.; SEBASTIÁN VICENTE, Ana (Ed.).

Patrimonio y etnografía de la escuela en España y Portugal durante el siglo XX. Murcia: Sociedad Española para el Estudio del Patrimonio Histórico-Educativo (Sephe); Centro de Estudios sobre la Memoria Educativa (Ceme)/Universidad de Murcia. 2012.

MORUS, Iwan Rhys.

Placing performance. Isis, v.101, n.4, p.775-778. 2010.

MORUS, Iwan Rhys.

The two cultures of electricity: between entertainment and edification in Victorian science. Science and Education, v.16, n.6, p.593602. 2006.
MOSLEY, Adam.

Objects, texts and images in the history of science. Studies in History and Philosophy of Science, v.38, n.2, p.289-302. 2007.

PAEDAGOGICA...

Paedagogica Historica: International Journal of the History of Education, v.40, n.1-2, p.9-227.

Disponível em: <https://www.tandfonline.com/ toc/cpdh20/40/1-2?nav=tocList $>$. Acesso em: 10 jul. 2019. 2004.

\section{PORTUGAL.}

Secretaria d'Estado dos Negócios do Reino.

Decreto de 17 de novembro de 1836. Plano dos Liceus Nacionais. Diário do Governo, n.275. 17 nov. 1836.

PROWN, Jules D.

Material/culture: can the farmer and the cowman still be friends? In: Kingery, David W. (Ed.). Learning from things: method and theory of material culture studies. Washington, DC: Smithsonian Institution Press. p.19-27. 1996.

RUDOLPH, John L.

Historical writing on science education: a view of the landscape. Studies in Science Education, v.44, n.1, p.63-82. 2008.

RUDOLPH, John L.

Turning science to account: Chicago and the general science movement in secondary education, 1905-1920. Isis, v.96, n.3, p.353-389. 2005.

RUDOLPH, John L.

Scientists in the classroom: the Cold War reconstruction of American science education. New York: Palgrave Macmillan. 2002.

SACCHETTO, Pier Paolo.

El objeto informador: los objetos de la escuela: entre la comunicación y el aprendizaje. Barcelona: Gedisa. 1986.

SHAPIN, Steven.

The House of Experiment in seventeenthcentury England. Isis, v.79, n.3, p.373-404. 1988.

SIMÓN CASTEL, Josep.

Les colleccions de física i química dels instituts de secundària: catalogació, estudi i metodologies. Actes d'història de la ciència i de la tècnica, v.1, n.1, p.85-94. 2008.

SIMÓN CASTEL, Josep.

Els instruments científics de l'IES Lluís Vives: primers resultats d'un catàleg de la cultura material de la ciência. Dissertação (Estudos Avançados em História da Ciência) - Universitat de Valencia, Valencia. 2002. 
SIMON, Josep.

Communicating science and pedagogy. In: Simon, Josep; Herran, Néstor (Ed.). Beyond borders: fresh perspectives in history of science. Newcastle: Cambridge Scholars. p.101-112. 2008.

SIMON, Josep.

Cataloguing manual 1.0. Valencia: Comissió d'Instruments Científics. 2006.

SIMON, Josep; HERRAN, Néstor (Ed.). Beyond borders: fresh perspectives in history of science. Newcastle: Cambridge Scholars Publishing. 2008.

SIMON, Josep; BERTOMEU SÁNCHEZ, José Ramón; GARCÍA BELMAR, Antonio.

Nineteenth-century scientific instruments in Spanish secondary schools. In: Lourenço, Marta C.; Carneiro, Ana (Ed.). The Laboratorio Chimico Overture: spaces and collections in the history of science, Lisbon. Lisboa: Museu de Ciência da Universidade de Lisboa (MCUL). p.167-184. 2009.

THE HISTORY...

The history of experimental science teaching. Science and Education, v.21, n.2, p.151-288.

[Thematic issue]. 2012.

VAN HELDEN, Albert; HANKINS, Thomas L. Introduction: instruments in the history of science. Osiris, v.9, p.1-6. 1994.

WARNER, Deborah Jean.

What is a scientific instrument, when did it become one, and why? British Journal of History of Science, v.23, n.1, p.83-93. 1990.

WARWICK, Andrew.

Masters of theory: Cambridge and the rise of mathematical physics. Chicago: The University of Chicago Press. 2003. 